\title{
El uso de beta bloqueantes luego de un infarto se asocia a menor mortalidad aún en los grupos en los que habitualmente se contraindica
}

Effect of beta blockade on mortality among high risk and low risk patients after myocardial infarction. S Gotlieb, R Mc Carter R Vogel. N Engl J Med 1998:339:489-97

\section{Objetivo}

Evaluar que pacientes se benefician con el tratamiento crónico con beta bloqueantes (BB) después de un infarto agudo de miocardio (IAM).

\section{Diseño}

Estudio de cohortes retrospectivo.

\section{Lugar}

Elevado número (no especificado) de hospitales de EE.UU que reportan sus egresos hospitalarios al sistema de MEDICARE (especie de PAMI de EE.UU.)

\section{Pacientes}

Fueron analizados un total de 201752 pacientes egresados con diagnóstico de IAM en el periodo 02/1994-07/1995.

\section{Medición de resultados principales}

Mortalidad en el seguimiento a dos años comparando pacientes con o sin tratamiento BB prescrito al alta hospitalaria. Dado que los grupos eran heterogéneos se ajustó el análisis para edad, grupo étnico, escore de Killip (insuficiencia cardíaca aguda), fracción de eyección, presión arterial, comorbilidad (diabetes, enfermedad pulmonar obstructiva crónica $[E P O C]$, asma), tipo de infarto ( $Q$ vs no $Q$ ), antecedentes y tratamiento concomitantes.

\section{Resultados principales}

La edad promedio fue 74 años, y $34,3 \%$ recibieron BB. Los pacientes que no recibieron $B B$ tenían mayor edad, clínica de insuficiencia cardíaca, y menor fracción de eyección, escore de APACHE II, y presión sistólica, todos parámetros fuertemente asociados con mayor mortalidad en el seguimiento. Cuando se realizó el análisis multivariable para evaluar el efecto independiente de los BB se observaron los resultados de la tabla.

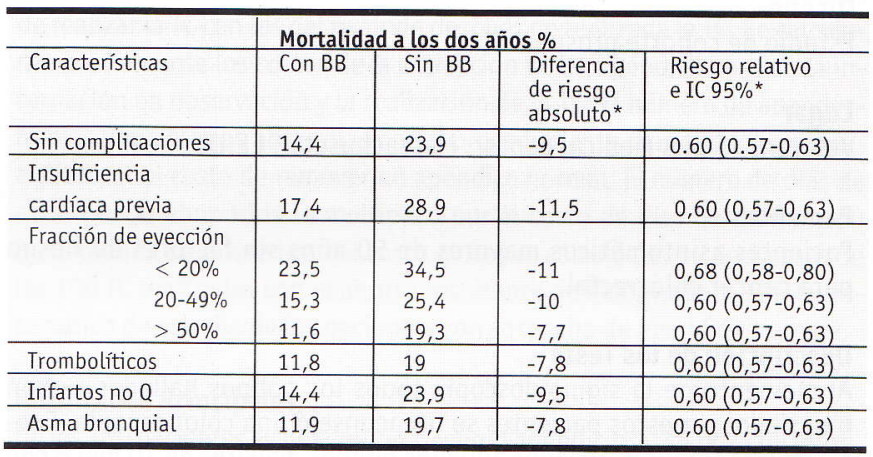

El beneficio se mantuvo con similar magnitud en todos los grupos etarios, aún en los mayores de 80 años, y fue más acentuado en los pacientes con EPOC.

\section{Conclusiones}

La mortalidad alejada luego de un IAM es menor en los pacientes tratados con BB, con una reducción de riesgo del $40 \%$. Este beneficio se observa aún en aquellas condiciones que habitualmente se consideran contraindicaciones como la edad avanzada, la insuficiencia cardíaca, la EPOC, así como también en indicaciones controvertidas como en el IAM no-Q.

\section{COMENTARIO}

Desde mediados de la década del 80 contamos con información definitiva generada por estudios aleatorizados de grandes dimensiones que han establecido la utilidad de la medicación BB después del IAM, en los pacientes que clínicamente pueden recibirlo ${ }^{1}$. El espectro de esta indicación se ha ampliado recientemente al comprobarse el beneficio sobre la morbimortalidad en la insuficiencia cardíaca en pacientes con cardiopatía isquémica. A pesar de eso, el uso de BB posIAM no se indica a la mayoría de los pacientes (en EE.UU. los reciben el $35 \%$, y el $52 \%$ en la red multicéntrica de la Sociedad Argentina de Cardiología²). Uno de los motivos es el prejuicio contra su uso en pacientes con daño ventricular aún sin insuficiencia cardíaca (es frecuente escuchar: hagamos un Ecocardio, si la función está deprimida evitemos los BB e indiquemos bloqueantes cálcicos, a pesar de que la evidencia indica reducción de la mortalidad con BB y probable aumento con bloqueantes cálcicos). ¿Qué nos aporta este estudio? Podemos adoptar dos actitudes alternativas frente a este diseño retrospectivo de cohorte: 1) Creer que el análisis estadístico permite comparar los grupos con exactitud a través de sus métodos de ajuste, $y$, por lo tanto, que los riesgos relativos son indicado- res de un real beneficio de los $B B$ en esta población. Esta actitud, que es la de los autores, contribuye a delimitar beneficio de los BB en grupos controvertidos, como los pacientes muy añosos, o EPOC, ambos excluidos de los estudios randomizados, o en los infartos no- $Q$ donde no se observó beneficio previamente. 2) Considerar que las poblaciones que reciben efectivamente $\mathrm{BB}$ tienen mucho menor riesgo, y que la reducción de riesgo relativo es una suma de un real beneficio, difícil de precisar con este diseño, y el menor riesgo basal de esta población. Dado que, por ejemplo, no se consideró enfermedad obstructiva vascular periférica, criterio de contraindicación de BB y asociado a un riesgo cinco veces mayor de muerte en el seguimiento en series previas, es cuestionable considerar que los ajustes matemáticos permiten homologar poblaciones retrospectivas seleccionadas para recibir o no BB por criterio médico. La mayor utilidad de este estudio es a mi juicio didáctica, aunque no de gran solidez científica: los BB no se usan con la frecuencia esperada en el postIAM, y pueden ser aplicados en una gran población con 'contraindicaciones' que no son tales. Para el cálculo del NNT, se sugiere utilizar los resultados del metaanálisis de los estudios randomizados.

*Ver Glosario

Dr. Carlos Daniel Tajer

Codirector de GEDIC. Grupo para el Estudio y la Docencia de la Investigación Clínica. Jefe de Cardiología. Sanatorio de la Providencia.

1. Yusuf S, Wittes J, Friedman J. Overview of results of randomized clinical trials in heart disease: I.Treatment following myocardial infarction. JAMA 1988:260:2088-2093.

2. Hirschon Prado A, Tajer C, Charask C y col. Comité de Investigación de la Sociedad Argentina de Cardiología y col. Encuesta Nacional de Infarto: evolución del tratamiento en los últimos diez años. Rev Argentina de Cardiol 1997:1:10-22. 